\title{
Wireless Heterogeneous Networks and Next Generation Internet
}

\author{
Eylem Ekici • Mehmet Can Vuran
}

Published online: 30 March 2010

(C) Springer Science+Business Media, LLC 2010

The recent advances in wireless access technologies as well as the increasing number of mobile applications have made Wireless Internet a reality. A wide variety of bandwidth demanding services including high speed data delivery and multimedia communication have been materialized through the convergence of the next generation Internet and heterogeneous wireless networks. However, providing even higher bandwidth and richer applications necessitates a fundamental understanding of wireless Internet architecture and the interactions between heterogeneous users. Consequently, fundamental advances in many concepts of the wireless Internet are required for the ultimate goal of communication anytime anywhere.

This special issue of the ACM Mobile Networks and Applications Journal is dedicated to the recent advances in the area of Wireless Internet. We accepted 10 papers out of 59 submissions from all over the world with a $17 \%$ acceptance rate. Papers describing management schemes, protocols, models, evaluation methods, and experimental studies of Wireless Internet are included in this special issue to provide a broad view of recent advances in this field.

In the first paper, R. Yu, Y. Zhang, M. Huang, and S. Xie address the call admission control (CAC) problem in cognitive radio networks in their work titled "Cross-Layer Optimized Call Admission Control in Cognitive Radio Networks." In addition to traditional CAC challenges, they

\footnotetext{
E. Ekici

Ohio State University,

Columbus, OH, USA

e-mail: ekici@ece.osu.edu

M. C. Vuran $(\bowtie)$

University of Nebraska-Lincoln,

Lincoln, NE, USA

e-mail: mcvuran@cse.unl.edu
}

also consider the presence of sensing errors that may mislead the CAC strategy to make an inefficient or even incorrect decision. To this end, the parameters of CAC strategy and spectrum sensing scheme are simultaneously tuned to minimize the dropping rate while satisfying the requirements of both blocking rate and interference threshold by the developed optimization framework. The crosslayer optimization is modeled as a non-linear programming problem after introducing a multiple-stair Markov model to approximate the non-memoryless state transitions. Accordingly, the dropping rate is shown to be reduced while still meeting the blocking rate and the interference probability constraints.

In their paper titled "Joint Dynamic Resource Allocation for QoS Provisioning in Multi-Access and Multi-Service Wireless Systems," D. Calabuig, J. F. Monserrat, D. Martín-Sacristán and N. Cardona analyze the resource allocation problem in multi-access wireless systems. The joint dynamic resource allocation (JDRA) algorithm simultaneously allocates the best-suited Radio Access Technology (RAT) and the amount of resources to all the users active in a multi-access wireless system. The Quality of Service (QoS) requirements of different users in terms of delay and bit rate are guaranteed through an optimization formalization based on a Hopfield Neural Network (HNN) formulation. The analysis and simulations highlight the advantages of joint decision approach compared to a twostep procedure and show that JDRA can address different levels of congestion and load distribution among RATs compared to the state-of-the-art.

In the paper titled "LAMA/CA: A Load-Adaptive MAC Protocol for Short Packets", Z. Noar develops a load adaptive medium access control protocol with collision avoidance. The protocol design is based on the fact that very often the Internet access messages, such as HTTP 
download requests, are based on short packets. Based on the traffic requirements, the MAC protocol is able to access to multiple overlapping base stations. Accordingly, the users do not have to use either collision detection or carrier sensing and whenever the number of colliding packets exceeds a pre-defined threshold, a congestion resolution algorithm is activated.

In their paper titled "Cross Layer Optimized Video Streaming based on IEEE 802.11 Multi-rate over Multi-hop Mobile Ad Hoc Networks", G. Lee and H. Song develop a cross-layer video streaming algorithm for communication over multi-hop mobile ad hoc networks. In general, video streaming service requires stringent quality of service requirements. The proposed algorithm aims to address the difficulties in guaranteeing the required QoS over MANET. According to the traffic requirements, the algorithm determines the modulation scheme at the physical layer, packet loss rate at the MAC layer, and media characteristics at the application layer. By adjusting the retransmission limit based on accumulated end-to-end delay, the perceptual video streaming quality is improved at the receiver.

The localization problem in wireless sensor and actor networks (WSAN) has been considered by G.A. Shah and O.B. Akan in their work entitled "Timing-based Mobile Sensor Localization in Wireless Sensor and Actor Networks." This problem is very important in WSANs as the performance of event detection and tracking highly depends on the exact location information of the sensors. Particularly, the problem becomes much more complicated when the sensor nodes as well as the anchor nodes (actors) are mobile. The authors address these challenges with a novel Timingbased Mobile Sensor Localization (TMSL) algorithm where, sensor nodes determine their distance from actors by using propagation time and speed of RF signal. To that end, actors actively broadcast reference beacons in a pattern of intervals adaptively defined according to the mobility of sensor nodes and the required level of localization accuracy. Although TMSL does not require any time synchronization among sensor nodes or with actors, the performance evaluations show that TMSL is adaptive to velocity of mobile sensor and actor nodes and can be configured according to the required localization accuracy to avoid overhead raised due to high velocity.

A decentralized multi-service group key management scheme is developed for wireless mesh networks by J. Hur and $\mathrm{H}$. Yoon in the paper titled "A Multi-service Group Key Management Scheme for Stateless Receivers in Wireless Mesh Networks". The scheme allows service providers to update and deliver their group keys to valid members in a distributed manner. Accordingly, the rekeying cost and storage overhead can be decreased for a member and a mesh point in multisender group communication environments. Through analysis they show that the pro- posed scheme guarantees backward and forward secrecy as well as group key secrecy against outside adversaries.

In their paper titled "An Experimental Study of the Performance Impact of Path-based DoS Attacks in Wireless Mesh Networks," A. K. Agarwal and W. Wang study the performance impact of path-based denial of service (DoS) attacks in wireless mesh networks. Through an experimental setup, they address the following questions: (1) How does the physical diversity affect the impact of path-based DoS attacks? (2) Are the medium errors conducive to pathbased attacks? (3) Is the collusion of attackers always helpful? and (4) Can there be a simple counter strategy to lessen the impact of such DoS attacks? To lessen the impacts of attacks in a WMN, a simple strategy is developed, where a helper node is placed in the network in such a way that it acts as an attacker to the attacker node, but does not impact the normal flows. Evaluations show that such a scheme can help subdue attackers' damage.

The problem of temporal fair scheduling has been investigated for queued data transmissions in wireless heterogeneous networks in the work entitled "Opportunistic Fair Scheduling in Wireless Networks: An Approximate Dynamic Programming Approach" by Z. Zhang, S. Moola, and E. K. P. Chong. The authors utilize a Markov decision process (MDP) with fairness constraints to formulate the transmission scheduling problem for throughput maximization and for delay minimization. The investigated fairness constraints include temporal as well as utilitarian fairness. Authors derive and prove explicit optimality equations for the above constrained MDPs, and give corresponding optimal fair scheduling policies. A practical stochasticapproximation-type algorithm and a novel approximation method have been presented, as well. Numerical results indicate significant performance improvements for both throughput maximization and delay minimization problems over other existing schemes.

The effects of a hierarchical architecture in peer-to-peer networks are investigated by Z. Ou, E. Harjula, T. Koskela and M. Ylianttila in their paper titled "GTPP: General Truncated Pyramid Peer-to-Peer Architecture over Structured DHT Networks." To this end, generalized multipletier hierarchical distributed hash table, called General Truncated Pyramid Peer-to-Peer (GTPP) architecture is developed.The results indicate that GTPP architecture decreases the expected lookup routing latency, improves the traffic distribution among all the peers, and can work with slightly lower maintenance traffic. The tradeoff comes as a higher expected lookup hop count that can be decreased closer to that of flat architectures by optimizing the sub-overlay setup. Tier counts of 2 to 3 are found to be most suitable in most cases for GTPP.

Finally, in their paper "On the Use of Ad Hoc Cooperation for Seamless Vertical Handoff and Its 
Performance Evaluation," H.-H. Choi and D.-H. Cho investigate the problem of vertical handoff based on the principle of ad hoc cooperation. A satisfactory solution to this problem is very important in heterogeneous network environments, where users must be provided with seamless service while satisfying quality-of-service constraints. In this paper, the authors propose a scheme where a neighbor ad hoc node assists some parts of the vertical handoff procedures requiring large latency, such as authentication and IP registration procedures. The paper details the vertical handover operation using the ad hoc cooperation and presents a performance evaluation.
Numerical results show that the proposed vertical handoff procedure decreases the service disruption time and the probability of packet loss, compared with the conventional handoff methods that do not consider cooperation.

We would like to thank the authors as well as the numerous reviewers who have contributed to the success of this special issue. We specially thank the Editor-in-Chief Prof. Imrich Chlamtac for giving us the opportunity to organize this special issue and his support and guidance throughout the organization process. We hope that you will enjoy reading this Special Issue. 